\title{
Molecular cloning and functional characterization of murine toll-like receptor 8
}

\author{
TINGTING LI, XIAOBING HE, HUAIJIE JIA, GUOHUA CHEN, SHUANG ZENG, \\ YONGXIANG FANG, QIWANG JIN and ZHIZHONG JING
}

\begin{abstract}
State Key Laboratory of Veterinary Etiological Biology,
Key Laboratory of Veterinary Public Health of Ministry of Agriculture, Lanzhou Veterinary Research Institute, Chinese Academy of Agricultural Sciences, Lanzhou, Gansu 730046, P.R. China
\end{abstract}

Received January 20, 2015; Accepted November 5, 2015

DOI: $10.3892 / \mathrm{mmr} .2015 .4668$

\begin{abstract}
Toll-like receptors (TLRs) are a large family of germ-line encoded pattern recognition receptors (PRRs) that recognize pathogen-associated molecular patterns and evoke the relevant innate immune responses. TLR8 is a member of several endosome nucleic acid-sensing TLRs; however little attention has been paid to murine TLR8 (mTLR8) compared with other endosome nucleic acid-sensing TLRs. In the present study, mTLR8 was cloned using reverse transcription-polymerase chain reaction from murine peripheral blood mononuclear cells and its function in regulating innate immune response was characterized. The open reading frame of mTLR 8 consists of 3,099 bps and encodes 1,032 amino acids. It contains typical leucine-rich repeats, a transmembrane domain and a Toll/interleukin-1 receptor domain, and it shares a high level of identity with other mammalian species. The expression of mTLR8 has been widely observed in different tissues, and higher expression levels of mTLR8 have mainly been detected in the heart, spleen and lung. Overexpression of MTLR8 is required for the activation of transcription factor nuclear factor- $\kappa \mathrm{B}$ and the production of tumor necrosis factor- $\alpha$. However, mTLR8 is not able to activate interferon regulatory factor 3 or activator protein 1 , nor can it induce interferon- $\alpha$ in HEK293T cells. These results indicate that mTLR8, as an important PRR, is indeed functional and is vital role in the activation of innate immune responses. This study may aid in determining the molecular basis of the interactions between mTLR8 and pathogens.
\end{abstract}

Correspondence to: Dr Zhizhong Jing, State Key Laboratory of Veterinary Etiological Biology, Key Laboratory of Veterinary Public Health of Ministry of Agriculture, Lanzhou Veterinary Research Institute, Chinese Academy of Agricultural Sciences, 1 Xujiaping, Lanzhou, Gansu 730046, P.R. China

E-mail: zhizhongj@163.com

Key words: mouse, toll-like receptor 8, cloning, expression analysis, innate immune response

\section{Introduction}

Toll-like receptors (TLRs), an important family of germ-line encoded pattern recognition receptors (PRRs), are responsible for the recognition of pathogen-associated molecular patterns (PAMPs) from infectious pathogens. Up to now, 10 TLRs (TLR1-10) have been identified in humans and 12 TLRs (TLR1-9, TLR11-13) in mice, and TLR1-9 have been found to be conserved in the two species (1). TLR1, 2, 4, 5 and 6 are primarily expressed on the surface and predominantly recognize bacterial and fungal cell wall components and viral envelope proteins, as well as protozoal components $(1,2)$. By contrast, the nucleic acid-sensing TLRs, which include TLRs $3,7,8,9$ and 13 , are localized within the endosomal compartments of immune cells and recognize double-stranded RNA (dsRNA), single stranded RNA (ssRNA) and DNA derived from viruses, bacteria, fungi and parasites (1-3). As an important family of type I transmembrane (TM) glycoprotein receptors, all TLRs are composed of an ectodomain (ECD) containing multiple leucine-rich repeats (LRRs) directly involved in the recognition of PAMPs, a TM domain required for the sub-cellular localization of TLRs, and an intracellular domain with a conserved cytoplasmic signaling region termed the Toll/IL-1 receptor (TIR), which is required for the transduction of downstream signaling (4). Upon PAMP recognition, the cytoplasmic TIR domain of the TLRs recruits the adaptor molecules myeloid differentiation primary response gene 88 (MyD88) and/or TIR-domain-containing adaptor-inducing interferon- $\beta$ (TRIF). This results in the activation of interferon regulatory factor 3 (IRF3), IRF7, activator protein 1 (AP-1) and nuclear transcription- $\mathrm{\kappa B}(\mathrm{NF}-\mathrm{\kappa B})$, as well as the transcription of inflammatory cytokines, chemokines, and type I interferons (IFNs) that rapidly initiate innate immune responses to ensure host protection (5).

The human TLR8 (hTLR8) gene is located on the X chromosome, and can recognize ssRNA, short dsRNA, bacterial RNA, oligoribonucleotides and a large number of synthetic chemical agonists, such as imidazoquinolines (1-4). However, although human TLR8 is able to recognize imidazoquinolines and initiate immune responses, murine TLR8 (mTLR8) is not activated by imidazoquinolines or ssRNA due to a five amino-acid deletion in the ECD. As a result, mTLR8 was 
initially hypothesized to be non-functional (1-4,6). However, in 2006 it was revealed that mTLR 8 could be activated by imidazoquinoline 3M-002 combined with poly(dT)17 oligonucleotides (ODNs), leading to NF- $\mathrm{B}$ activation and TNF- $\alpha$ production (7), suggesting that mTLR8 is indeed functional. In support of this, a recent study reported that vaccinia virus (VACV) or vaccinia viral poly(A)/T-rich DNA could activate $\mathrm{NF}-\kappa \mathrm{B}$ in an mTLR8-dependent manner. In addition, synthetic poly(dA) and poly(dT) ODNs are capable of activating plasmacytoid DCs (pDCs) in an mTLR8-dependent manner (8), suggesting that mTLR8 is a functional receptor, regulating innate immunity against VACV infection. However, research from another team has raised uncertainties about these results, not only because they found that poly A10 and two different polyT ODNs did not induce IFN- $\alpha$ or other cytokines in sorted FL-pDCs or ex vivo-isolated pDCs, but also because of the high levels of transcripted TLR7 and TLR9 in murine pDCs and not TLR8 (9). Notably, mTLR8 was found to inhibit TLR7-sensing of 3M-001 in HEK293T cells, and mTLR8 ${ }^{-1-}$ DCs showed increased responses to various TLR7 ligands and NF- $\kappa \mathrm{B}$ activation. These results indicate that TLR8 may directly modulate TLR7 function (10).

In this study, to investigate the role of mTLR8 in regulating innate immune responses, mTLR8 cDNA isolated from peripheral blood mononuclear cells (PBMCs) was cloned and sequenced. It was found that mTLR8 conserved the typical domains of TLRs and had a high level of identity to other mammalian species. Higher expression levels of mTLR8 in the heart, spleen, and lung were also detected by reverse transcription-quantitative polymerase chain reaction (RT-qPCR). Furthermore, it was demonstrated that mTLR8 can induce nuclear factor $(\mathrm{NF})-\kappa \mathrm{B}$ activation and tumor necrosis factor (TNF)- $\alpha$ production but not the activation of interferon-sensitive response element (ISRE), AP-1 activation and IFN- $\alpha$ production in HEK293T cells. Overall, these results provide a molecular foundation for further investigation into the potential role of mTLR 8 in anti-viral therapeutics, oncotherapy, autoimmune diseases and vaccine design.

\section{Materials and methods}

Animals and cells. C57BL/6 mice $(\mathrm{n}=30)$ were purchased from the Laboratory Animal Center of Lanzhou University (Lanzhou, China). Groups of 6-10-week-old mice were selected for this study (Animals were treated in accordance with the Guide for the Care and Use of Laboratory Animals and approved by the Committee of Transgenic Bio-safety Evaluation of Agriculture Ministry, Lanzhou, China). Animals were housed separately under $12 \mathrm{~h}$ light/dark cycles at a temperature of $22^{\circ} \mathrm{C}$ and a humidity of $60 \%$, with free access to food and water. HEK293T cells were purchased from the Type Culture Collection of the Chinese Academy of Science (Shanghai, China) and were maintained in complete Dulbecco's modified Eagle's medium (Gibco, Thermo Fisher Scientific Inc., Waltham, MA, USA) supplemented with $10 \%$ fetal bovine serum (Gibco, Thermo Fisher Scientific Inc.), and $50 \mathrm{mg} / \mathrm{ml}$ penicillin/streptomycin (Shanghai Sangon Biological Engineering Biotechnology Company, Shanghai, China).
Tissue sample collection and total RNA isolation. The mice were sacrificed by enucleation of the eye using $2 \%$ pentobarbital sodium (Wuhan Kehaojia Biological Technology, Wuhan, China) and blood samples were collected for mononuclear cell isolation using lymphocyte separation medium (Sigma-Aldrich, St. Louis, MO, USA). The heart, liver, spleen, lung, kidney, intestine and muscle were dissected, washed three times in phosphate-buffered saline (PBS, pH 7.2) and immediately snap-frozen in liquid nitrogen prior to being stored at $-80^{\circ} \mathrm{C}$ until required. Total RNA was extracted from the collected tissue samples and cells using Takara MiniBEST Universal RNA Extraction kit (Takara, Dalian, China) according to the manufacturer's instructions. The RNA quality was detected by $1 \%$ agarose gel electrophoresis (Shanghai Sangon Biological Engineering Biotechnology Company) which was stained with $10 \mu \mathrm{g} / \mathrm{ml}$ ethidium bromide (Shanghai Sangon Biological Engineering Biotechnology Company). The total RNA concentration was determined using a NanoDrop 2000 spectrophotometer (Thermo Fisher Scientific, Inc.) and the optical density (OD)260:OD280 ratio of the RNA was between 1.8 and 2.0 .

Reverse transcription. Synthesis of the first strand of cDNA was performed with the Primescript 1st strand cDNA synthesis kit (Takara Bio Inc., Dalian, China) according to the manufacturer's instructions. Briefly, $800 \mathrm{ng}$ RNA, $0.5 \mu \mathrm{l}$ Oligo dT Primer $(50 \mu \mathrm{M}), 0.5 \mu \mathrm{l}$ random 6 mers $(50 \mu \mathrm{M}), 1 \mu \mathrm{l} \mathrm{dNTP}$ mixture $(10 \mathrm{mM})$ and an appropriate volume of RNase-free water up to $10 \mu \mathrm{l}$ were mixed gently. The mixture was heated to $65^{\circ} \mathrm{C}$ for $5 \mathrm{~min}$, and then quick-chilled on ice. Then, the reverse transcription mixture was prepared as follows: $4 \mu \mathrm{l}$ 5X PrimeScript Buffer, $0.5 \mu \mathrm{l}$ RNase inhibitor (40 U/ $\mu \mathrm{l}), 1 \mu \mathrm{l}$ PrimeScriptRTase $(200 \mathrm{U} / \mu \mathrm{l})$ and $4.5 \mu \mathrm{l}$ RNase free water were mixed with the above mixture. Cycle parameters of the RT procedure were 1 cycle of $30^{\circ} \mathrm{C}$ for $10 \mathrm{~min}, 42^{\circ} \mathrm{C}$ for $60 \mathrm{~min}$, and $95^{\circ} \mathrm{C}$ for $5 \mathrm{~min}$. The cDNA were stored at $-80^{\circ} \mathrm{C}$ for mTLR8 cloning and relative quantification by PCR.

Cloning of murine TLR8 cDNA and construction of pCMV-tag2B-TLR8 eukaryotic expression vector. Based on the murine genomic sequence obtained from the BLAST search, the primers c-mTLR8-F and c-mTLR8-R (Table I) were designed, and used to amplify the potential mTLR8cDNA by reverse transcription-PCR from total RNA extracted from PBMCs. A pair of primers (c-mTLR8) were designed based on the coding sequence (CDS) of Mus musculus TLR8 (GenBank ID: NM_133212) with Primer Premier 5.0 and with XhoI and $K p n I$ site (underlined). The PCR was performed in a $50 \mu 1$ reaction volume containing $4 \mu \mathrm{l}$ of the first-strand cDNA, $3 \mu \mathrm{l}$ each of forward and reverse primers $(10 \mu \mathrm{M}), 10 \mu \mathrm{l} 5 \mathrm{X}$ PrimeSTAR Buffer (including $\mathrm{Mg}^{2+}$; Takara Bio Inc.), $4 \mu \mathrm{l}$ dNTP mixture, $0.5 \mu 1$ PrimeSTAR HS DNA Polymerase (Takara Bio Inc.) and $25.5 \mu \mathrm{l}$ sterile water. PCR conditions were as follows: Denaturation at $95^{\circ} \mathrm{C}$ for $4 \mathrm{~min}, 30$ cycles at $98^{\circ} \mathrm{C}$ for $10 \mathrm{sec}, 64^{\circ} \mathrm{C}$ for $15 \mathrm{sec}, 72^{\circ} \mathrm{C}$ for $3 \mathrm{~min} 40 \mathrm{sec}$, with a final extension of $72^{\circ} \mathrm{C}$ for $10 \mathrm{~min}$.

The PCR products were analyzed by electrophoresis on $1.0 \%$ agarose gel with $10 \mu \mathrm{g} / \mathrm{ml}$ ethidium bromide, and purified by a gel extraction kit (Axygen Scientific Inc., Union City, CA, USA). The purified products and the vector pCMV-Tag2B 
Table I. Primers used for reverse transcription-quantitative polymerase chain reaction.

\begin{tabular}{|c|c|c|c|c|}
\hline Gene & Primer & Sequence $\left(5^{\prime}-3^{\prime}\right)$ & $\begin{array}{l}\text { Amplicon } \\
\text { length (bp) }\end{array}$ & $\begin{array}{c}\text { GenBank } \\
\text { accession no. }\end{array}$ \\
\hline q-mTLR8 & $\begin{array}{l}\text { Forward } \\
\text { Reverse }\end{array}$ & $\begin{array}{l}\text { ACCTGAGCCACAATGGCATTTAC } \\
\text { TTGCCATCATTTGCATTCCAC }\end{array}$ & 121 & NM_133212 \\
\hline$\beta$-actin & $\begin{array}{l}\text { Forward } \\
\text { Reverse }\end{array}$ & $\begin{array}{l}\text { CATCCGTAAAGACCTCTATGCCAAC } \\
\text { ATGGAGCCACCGATCCACA }\end{array}$ & 171 & NM_007393 \\
\hline c-mTLR8 & $\begin{array}{l}\text { Forward } \\
\text { Reverse }\end{array}$ & $\begin{array}{l}\text { TTTCTCGAGATGGAAAACATGCCCCCTCAGTC } \\
\text { CGGGGTACCCTAGTATTGCCTAATGGAATCAATG }\end{array}$ & 3099 & NM_133212 \\
\hline mTNF- $\alpha$ & $\begin{array}{l}\text { Forward } \\
\text { Reverse }\end{array}$ & $\begin{array}{l}\text { CAGGGTACCGTTTTCCGAGGGTTGAATGAG } \\
\text { CAGCTCGAGGTCTTTTCTGGAGGGAGATGTG }\end{array}$ & 243 & GQ917239 \\
\hline mIFN- $\alpha 2$ & $\begin{array}{l}\text { Forward } \\
\text { Reverse }\end{array}$ & $\begin{array}{l}\text { CCGGGTACCCAGAGAGTGAAGTAAAGAAAGTG } \\
\text { TGTCTCGAGTGTGGGTCTTGCAGAGGTTGAT }\end{array}$ & 180 & X01969 \\
\hline
\end{tabular}

CTCGAG refers to XhoI site and GGTACC refers to KpnI site. TLR8, toll-like receptor 8; TNF, tumor necrosis factor.

(Stratagene, La Jolla, CA, USA) were digested by XhoI and $K p n I$ (Takara Bio Inc.) and the products were purified by electrophoresis and a gel extraction kit. The digested PCR product was inserted into the vector pCMV-Tag2B. Proper construction was confirmed by sequencing and digesting with XhoI and KpnI and was termed pCMV-Tag2B-mTLR8.

Sequence, structure and phylogenetic analysis. Amino acid sequences were aligned using the Clustal Omega online sequence alignment tool (http://www.ebi. ac.uk/Tools/msa/clustalo/) and the Sequence Manipulation Suite (SMS; http://www.bio-soft.net/sms/), and the phylogenetics of the molecular evolutionary analysis were conducted using the MEGA 5.1 program (http://www.megasoftware.net/). Prediction of the mTLR8 and hTLR8 domains, motifs and features was performed on the SMART website (http://smart. embl-heidelberg.de/). Swiss-model (http://swissmodel.expasy. org/) was used to simulate the crystal structure of mTLR8. The predicted structure was analyzed and compared to that of hTLR8 (PDB ID: 3w3 g) using Swiss-pdb Viewer 4.0.1 software (Swiss Institute of Bioinformatics).

Construction of reporter plasmids, transfection and reporter luciferase assays. Murine TNF- $\alpha$ promoter region (GenBank ID: GQ917239) and IFN- $\alpha 2$ promoter region (GenBanK ID: X01969) was amplified from murine genomic DNA, which was extracted from murine splenic lymphocytes, and then cloned into the pGL4.10 basic vector capable of expressing firefly luciferase. The primers are shown in Table I with KpnI and XhoI sites (underlined). HEK293T cells were seeded at a density of $2 \times 10^{5}$ cells/well in $500 \mu \mathrm{l}$ culture medium into 24 -well plates and incubated. LyoVec (25 $\mu \mathrm{l}$; Invivogen, San Diego, CA, USA) was brought to room temperature and gently vortexed to homogenize, after which it was mixed gently with $0.5 \mu \mathrm{g} /$ well of pCMV-Tag2B-mTLR8 plasmid (pCMV-Tag2B empty plasmid as controlled), $0.2 \mu \mathrm{g}$ /well of the reporter plasmids including pGL4.32 [luc $2 \mathrm{P} / \mathrm{NF}-\kappa \mathrm{B}-\mathrm{RE} / \mathrm{Hygro}]$ vector, pGL4.44 [luc2P-AP1-RE-Hygro] vector, pGL4.45
[luc2P-ISRE-Hygro] vector (Promega Corporation, Madison, WI, USA), TNF- $\alpha$-Luc and IFN- $\alpha 2$-Luc, and $0.01 \mu \mathrm{g} /$ well pGL4.74 [hRluc/TK] vector (Promega Corporation), in a sterile $0.5 \mathrm{ml}$ microfuge tube. When the cells had grown to $70-90 \%$ confluency, the culture medium was gently replaced with $475 \mu 1$ opti-MEM (Gibco, Thermo Fisher Scientific Inc.) and $25 \mu 1$ LyoVec-DNA complexes were directly added to the medium. The mixture was agitated to distribute the complexes uniformly and incubated for $24 \mathrm{~h}$ for the subsequent assay. After a 24-h transfection, the dual luciferase reporter system was used to detect the luciferase activity (Promega Corporation), according to the manufacturer's protocol. The reporter assays were repeated three times in duplicate.

Tissue distribution of murine TLR8 detected by RT-qPCR. Primers for mTLR8 (q-mTLR8) were designed based on the Mus musculus TLR8 cDNA sequence and $\beta$-actin was used as an internal reference gene to normalize target gene transcript levels (Table I). Quantitative PCR was performed with the Mx3005P Real-time PCR detection system (Agilent Technologies Deutschland GmbH, Waldbronn, Germany). PCR was performed in $20 \mu \mathrm{l}$ reactions with $2 \mu \mathrm{l}$ of the cDNA sample, $0.8 \mu \mathrm{l}$ each of the forward and reverse primers $(10 \mu \mathrm{M})$, $6.4 \mu 1$ DEPC-treated water, and $10 \mu 1$ 2xSYBR Premix Ex Taq II (Takara Bio Inc.). The PCR parameters include $95^{\circ} \mathrm{C}$ for $30 \mathrm{sec}, 40$ cycles at $95^{\circ} \mathrm{C}$ for $5 \mathrm{sec}$ and $60^{\circ} \mathrm{C}$ for $34 \mathrm{sec}$, and then $95^{\circ} \mathrm{C}$ for $15 \mathrm{sec}, 60^{\circ} \mathrm{C}$ for $1 \mathrm{~min}$, and $95^{\circ} \mathrm{C}$ for $15 \mathrm{sec}$. The primers for TLR8 and $\beta$-actin yielded a single peak in the melting curve and a single band of the expected size on an agarose gel. Data were analyzed according to the efficiency-corrected comparative $\mathrm{Cq}$ method and normalized using $\beta$-actin expression levels.

Statistical analysis. All results represent the mean of three separate experiments. RT-qPCR data are expressed as the mean \pm standard deviation. The statistical significance of differences was assessed using Student's t-test. $\mathrm{P}<0.05$ was considered to indicate a statistically significant difference. 


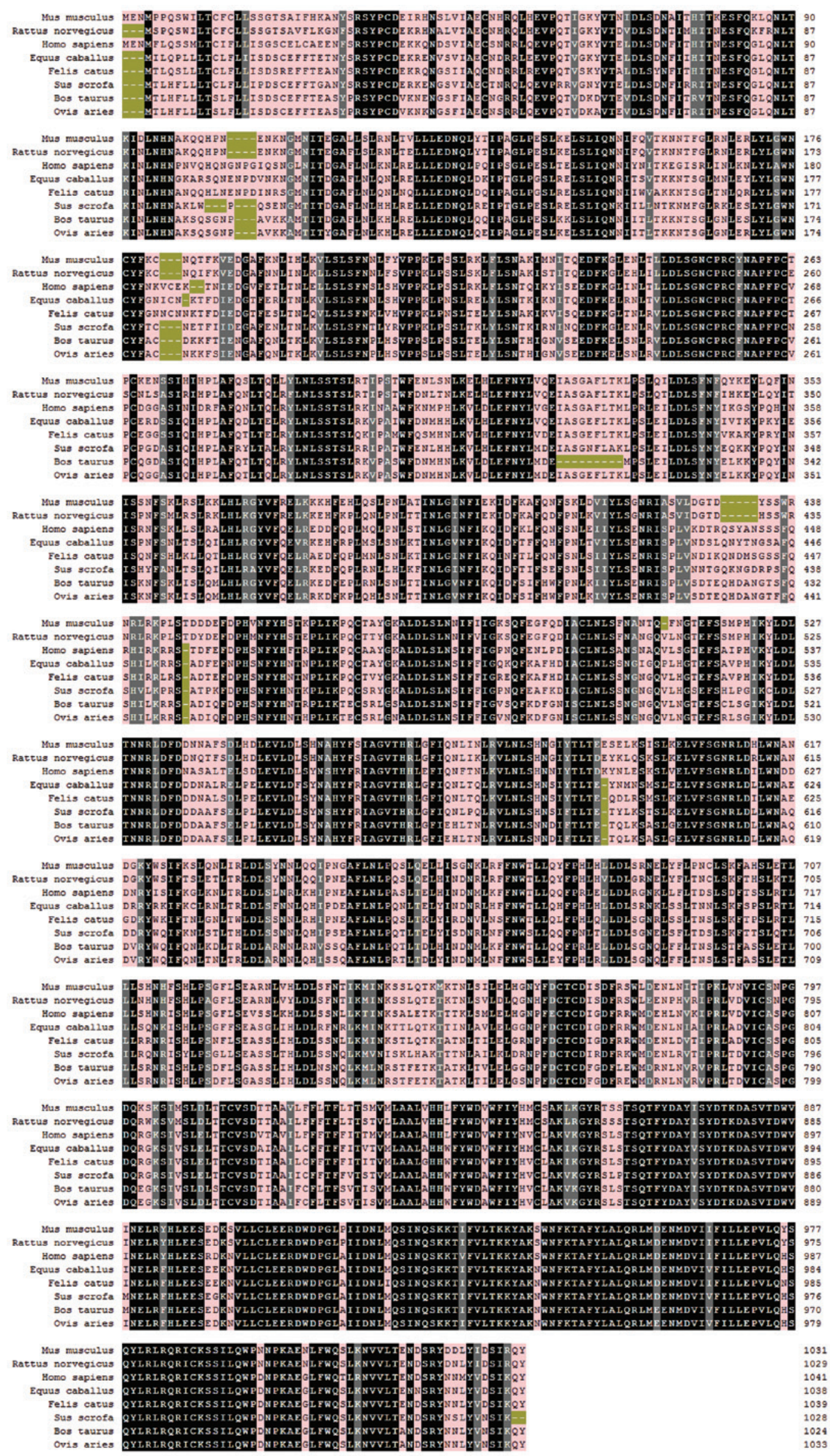

Figure 1. Sequence analysis of murine TLR8. Alignment of amino acid sequence of murine TLR8 with TLR8 of seven other species: Rattus norvegicus, Homo sapiens, Equus caballus, Felis catus, Sus scrofa, Bos taurus and Ovis aries. Black represents the same amino acids; dark gray represents similar amino acids; light red represents different and similar amino acids; dark yellow represents an amino acid gap. TLR8, toll-like receptor 8 . 


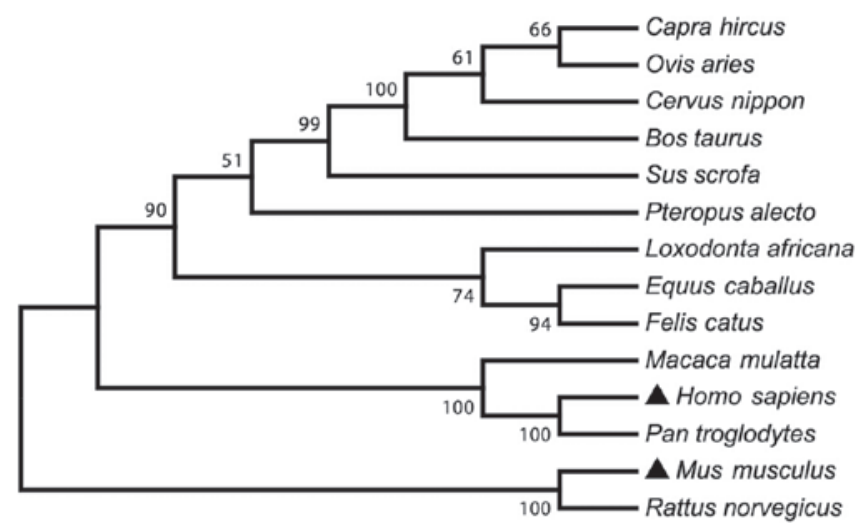

Figure 2. A phylogenetic tree of the amino acid sequences of TLR8 in 14 mammalian species. The sequences were obtained from GenBank with accession numbers: NP_001029109 (Bos taurus), NP_001272686 (Capra hircus), ADZ17145 (Cervus nippon), NP_001104771 (Equus caballus), ABS28967 (Felis catus), NP_619542 (Homo sapiens), ABS28968 (Loxodonta africana), NP_001123899 (Macaca mulatta), AAK62677 (Mus musculus), NP_001129401 (Ovis aries), NP_001123944 (Pan troglodytes), ADO01610 (Pteropus alecto), ABM92444 (Rattus norvegicus) and NP_999352 (Sus scrofa). The unrooted tree was built using ClustalX by neighbour-joining method. Bootstrap values are indicated on the branches. TLR8, toll-like receptor 8.

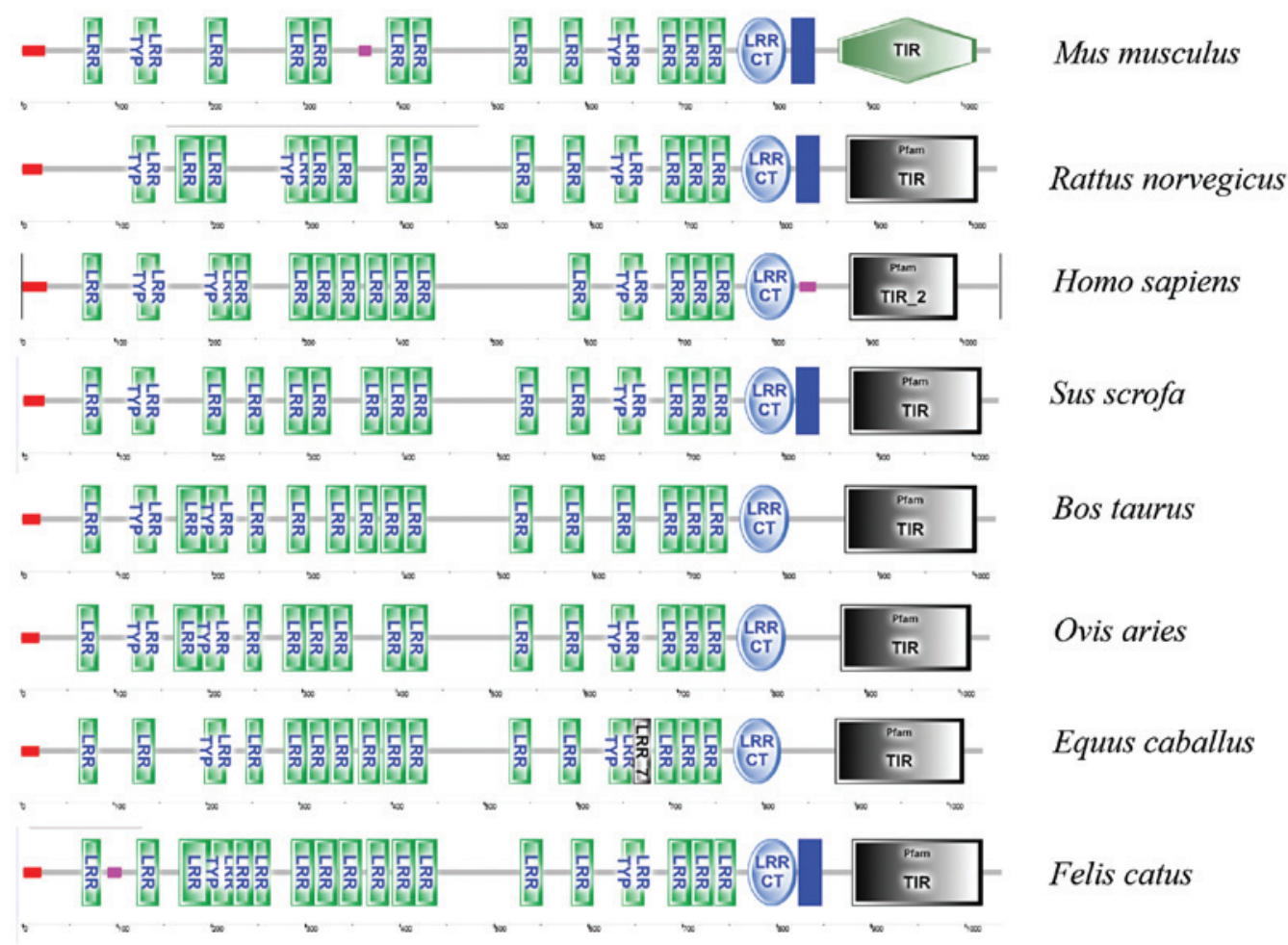

Figure 3. Predicted secondary structures of TLR8 by SMART including Mus musculus, Rattus norvegicus, Homo sapiens, Sus scrofa, Bos taurus, Ovis aries, Equus caballus and Felis catus. TLR8, toll-like receptor 8; LRR, leucine-rich repeats; TYP, typical; CT, C-terminal; TIR, Toll/IL-1 receptor.

\section{Results}

Cloning, sequence and structure analysis of murine TLR8. Based on the murine genomic sequence obtained from the BLAST search, the primers c-mTLR8-F and c-mTLR8-R (Table I) were designed and used to amplify the potential mTLR8cDNA by RT-PCR from total RNA extracted from PBMCs. The open reading frame (ORF) of mTLR8 is 3,099 bps and it encodes 1,032 amino acid residues. It was determined that the nucleotide sequence recorded for mTLR8 was completely identical to that held in the NCBI (GenBank ID: NM_133212, AY035890.1 and BC132054.1) (Fig. 1).
Multiple sequence alignment against other TLR8 sequences showed that the nucleotide sequence of MTLR8 was $91.1 \%$ identical to that of rat TLR8, 76.1\% identical to that of human TLR8, 73.0\% identical to that of bovine TLR8, 73.5\% identical to that of ovine TLR8, 76.7\% identical to that of equine TLR8, $76.0 \%$ identical to that of feline TLR8 and $74.0 \%$ identical to that of porcine TLR8. At the amino acid level, the levels of identity were $88.2,70.9,67.3,68.8,72.1,71.6$ and $69.6 \%$, respectively (Fig. 1). In addition, phylogenetic analysis showed that mTLR8 is closely associated with rat, compared with other species in genetic evolution. Therefore, mTLR8 was more diverse from TLR8 from other species (Fig. 2). 

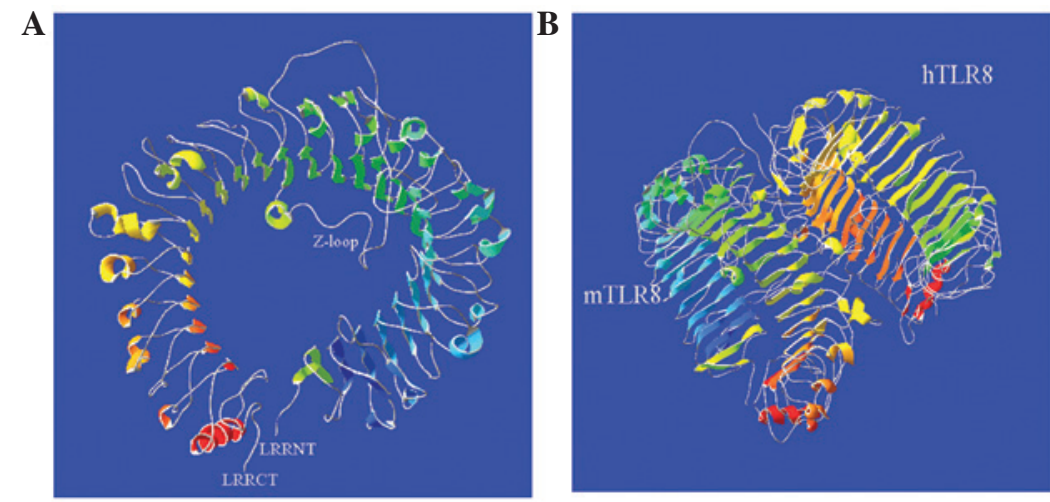

Figure 4. (A) Mimetic crystal structure of murine TLR8 LRRs by a Swiss-model. (B) Structure comparison of murine TLR8 (left) and hTLR8 (right). TLR8, toll-like receptor 8. LRR, leucine-rich repeat; CT, C-terminal; NT, N-terminal.

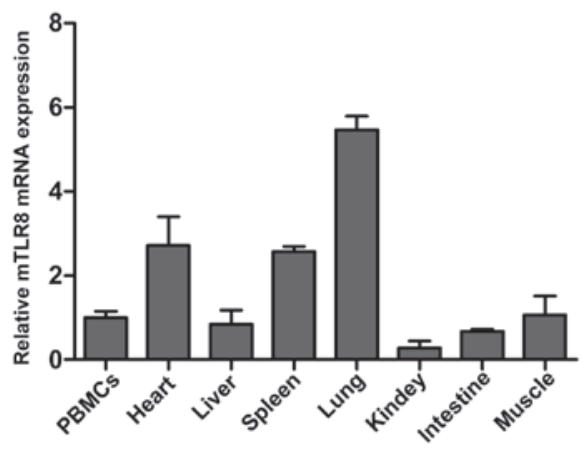

Figure 5. Relative expression levels of mTLR8 mRNA transcripts in murine tissues. Reverse transcription-quantitative polymerase chain reaction was performed to measure the expression level in PBMCs and heart, liver, spleen, lung, kidney, intestinal and muscle tissues. Data were analyzed according to the efficiency-corrected comparative $\mathrm{Cq}$ method and normalized using $\beta$-actin expression levels. Data were normalized to the PBMCs and error bars indicate standard deviation. All RNA samples were free of genomic contamination after DNase I (RNase free) treatment. PBMCs, peripheral blood mononuclear cells; TLR8, toll-like receptor 8 .

To predict the structural domains in mTLR8, the putative amino acid sequences were analyzed using the SMART program. As shown in Fig. 3, mTLR8 contains features common to TLRs, namely multiple extracellular LRR, a TM domain and an intracellular TIR domain. Additionally, similar domains can be predicted in TLR8 from bovine, equine and canine, indicating that the ligand-binding domain may be a common characteristic of TLRs in ligand recognition among a number of species (Fig. 3). Other domains with high inter-species conservation included the $\mathrm{N}$ - and $\mathrm{C}$-terminal cysteine-flanked LRRs, as well as the TM and TIR domains (Fig. 3). Moreover, the LRRs of mTLR8 displayed a space structure like a solenoid coil and contained $38 \beta$-ovinets located on its concave surface and $23 \alpha$-helixes on the convex side (Fig. 4A). Compared with the structure of hTLR8, except for extra $7 \alpha$-helixes, no differences between mTLR 8 and hTLR8 were identified, suggesting that their crystal structures were almost identical (Fig. 4B).

Expression of murine TLR8 in different tissues. The mRNA expression profile of mTLR8 mRNA in different tissues was analyzed by RT-qPCR using the $\beta$-actin housekeeping gene as a control. As shown in Fig. 5, mTLR8 mRNA was expressed at a
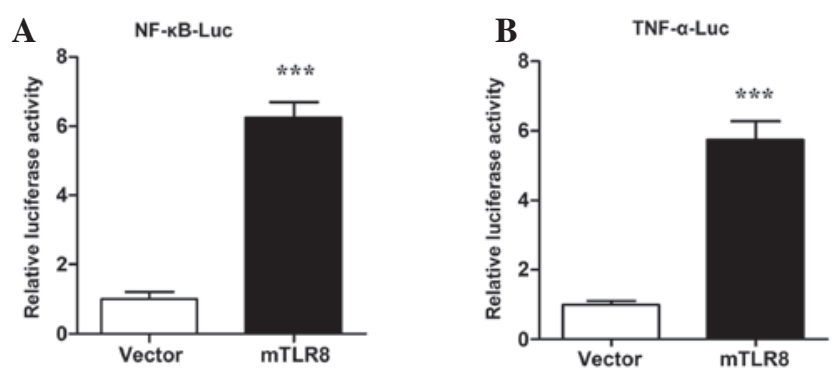

C
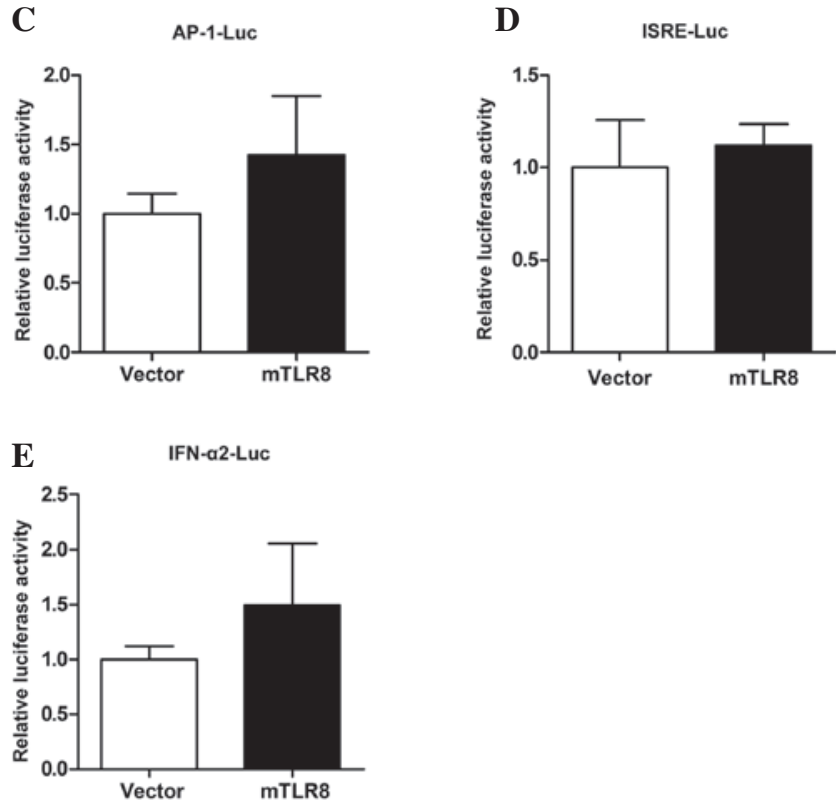

Figure 6. Murine TLR8 stimulates NF- $\kappa \mathrm{B}$ to induce TNF- $\alpha$ expression in HEK293T cells. HEK293T cells were co-transfected with $500 \mathrm{ng} /$ well expression plasmids (mTLR8 or empty vector) together with $200 \mathrm{ng} /$ well of a reporter

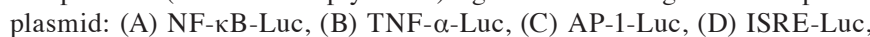
(E) IFN- $\alpha 2$-Luc, along with $10 \mathrm{ng} /$ well phRL-TK using LyoVec. Luciferase activity of NF- $\mathrm{BB}$ was quantified with the Dual-Glo Luciferase Assay kit $24 \mathrm{~h}$ after transfection. The reporter assays were repeated three times in duplicate. ${ }^{* * *} \mathrm{P}<0.001$ as compared with the vector. TLR8, toll-like receptor 8 ; $\mathrm{NF}$, nuclear factor; TNF, tumor necrosis factor; AP-1, activator protein 1; ISRE, interferon-sensitive response element.

detectable level in all the monitored tissues, including PBMCs, heart, liver, spleen, lung, kidney, small intestine and muscle. Notably, expression was most abundant in the lung, followed 
by the heart and spleen, with somewhat less in PBMCs, liver, small intestine, muscle and kidney. This indicated differences in the expression levels of mTLR8 between different murine tissues. The biological implications of the varying levels of expression in differing tissues in mice, particularly in immune cells (including pDCs and cDCs), is unclear and requires further investigation $(9,10)$. In humans, the most abundant expression of TLR8 has been found in the lung and PBMCs, indicating that the expression profiles of mTLR8 in tissue are similar to hTLR8 $(11,12)$.

Functional analysis of murine TLR8. To investigate whether mTLR8 is able to activate the signal pathways of innate immunity, HEK293T cells were transfected with an mTLR8 expression construct together with murine NF- $\kappa \mathrm{B}-$, ISRE-, AP-1-, TNF- $\alpha$ - and IFN- $\alpha 2$-luciferase reporter plasmids. The dual luciferase report system was used to record the activity of the luciferase reporter plasmid. As shown in Fig. 6A and B, $\mathrm{NF}-\kappa \mathrm{B}$ and TNF- $\alpha$ promoter activity was significantly activated in cells over-expressing mTLR8 compared with cells transfected with the empty vector $(\mathrm{P}<0.001)$. A study has revealed that mTLR8 induced NF- $\kappa \mathrm{B}$ activation and TNF- $\alpha$ production in murine PBMCs and mTLR8-transfected HEK293 cells (7). In order to further explore whether mTLR8 could activate other regulatory elements or promote type I IFNs, the luciferase activity of the ISRE, AP-1 and IFN- $\alpha 2$ promoters was also investigated. The results showed that over-expression of mTLR8 in the HEK293T cells did not activate ISRE, AP-1, or IFN- $\alpha 2$ (Fig. 6C-E). Therefore, these results showed that over-expression of mTLR 8 can activate NF- $\kappa \mathrm{B}$ and TNF- $\alpha$ but does not have the ability to induce the activation of IRF3, AP-1 and IFN- $\alpha 2$ in the HEK293T cells.

\section{Discussion}

As an important member of the endosomal TLRs, mTLR8 was hypothesized to be non-functional as it was initially observed that $\mathrm{TLR}^{-/-}$mice are unresponsive to the TLR7/8 agonist R848, or TLR8 RNA ligands, even though human TLR8 and mTLR8 are close relatives (13-15). However, recent studies have revealed that mTLR8 can be activated and is important in controlling TLR7 expression (10). In the present study, mTLR8 was cloned and characterized. The results clearly show that mTLR8 is an important endosomal TLR and is critical in the innate immune signaling pathway.

The ORF of mTLR8 comprises 3,099 bps, encoding a 1,032-amino-acid polypeptide. In addition to human and mTLR8, the TLR8 sequences from rats (ABM92444), bovine (NP_001029109), ovine (NP_001129401), equines (NP_001104771), cats (ABS28967), porcines (NP_999352), and other mammalian species have already been identified. Notably, mTLR8 is more similar to rat TLR8 at the nucleotide and amino acid level, compareed with other mammalian species, suggesting mTLR8 is also conserved in the process of evolution and the mTLR8 acts as a good model to research the innate immune system of humans or other species. In addition, mTLR8 is also composed of LRRs, a TM domain and a TIR domain. High conservation of the three subsections of the TIR domain (boxes 1, 2, 3) known to be important in signaling (boxes 1 and 2) and receptor localization (box 3), indicate that the ligand-binding domain may be a common characteristic of TLRs in ligand recognition among a range of species (4). Furthermore, like the crystal structure of hTLR8, the extracellular domain of mTLR8 can also form a ring-shaped structure in which each half of the ring is produced by the $\mathrm{N}$-and C-terminal fragments, and this ring-shaped structure is different from the typical equineshoe-shaped structure exhibited by other TLRs $(4,16)$. Comparative analysis has found that MTLR8 and hTLR 8 have the same numbers of $\beta$-ovinets, spanning the whole concave face of the ring-shaped structure, as well as which, it features a Z-loop. Although there is no difference in the crystal structures of mTLR 8 and hTLR8, mTLR8 was unresponsive to hTLR8 ligand stimulation. A recent study has shown that a five-amino-acid-residue motif (RQSYA) that is conserved with varying sequencing in several non-rodent species, is absent in rodent species mTLR8 and that $\operatorname{rat}(\mathrm{r})$ TLR8 was not responsive to ligand stimulation in the absence of poly(dT) ODN (17). As shown in Fig. 1, mTLR8 also lacks a five-amino-acid motif (RQSYA) that is located in an undefined region following LRR-14 of the mTLR8 ECD. The Z-loop is located between LRR14 and LRR15 which may also be the location of the five-amino-acid motif (RQSYA). Thus, species-specific ligand recognition is predicted on the differences between the Z-loops of mTLR8 and hTLR8.

Expression of TLRs in different tissues is an important determinant of their function. Previous studies have revealed that hTLR8 is highly expressed in lung and PBMCs and modestly expressed in the spleen, lymph nodes, bone marrow and placenta $(11,12)$. In the present study, mTLR8 was expressed in all analyzed tissues, including PBMCs, heart, liver, spleen, lung, intestine and muscle, with the highest expression identified in the lung. However, it was modestly expressed in the heart and spleen, and rarely in the kidney, reflecting possible species-specific differences in the expression of TLR 8 between mice and humans. Notably, significant amounts of mTLR8 were detected in the heart, a non-immune tissue, that usually produces little TLR8 in either humans or mice. The biological implications of these different expression patterns of TLR8 in different tissues are unclear and require further investigation. In addition, although there are differences in the TLR8 mRNA expression profiles of the different species, abundant TLR8 mRNA expression can be observed in the lung, spleen, PBMCs, liver and lymph nodes of humans, mice and porcines, suggesting that TLR 8 is important in the mammalian immune system $(11,12,18)$. Furthermore, hTLR8 is primarily expressed in monocytes/macrophages and myeloid DCs, and predisposes the induction of inflammatory cytokines, whereas mTLR8 is selectively expressed in DCs derived from splenocytes, namely CD4 $4^{+} \mathrm{DCs}, \mathrm{CD} 8^{+} \mathrm{DCs}$, CD4-CD8-DCs and pDCs (19-21). However, similar to hTLR8, murine pDCs are also generally known to express TLR7 and TLR9, but not TLR8, however, that the precise expression of mTLR8 mRNA in pDCs and cDCs remains to be elucidated.

As an important PRR that is critical in virally induced type I IFNs and inflammatory cytokine expression, overexpression of hTLR8 can activate IRF3 and NF- $\kappa$ B and suppress viral replications. Previous studies have revealed that hTLR8 can recognize antiviral compounds, ssRNA, oligoribonucleotide and small interfering RNA (1-4,6). In addition, bovine and porcine TLR8 are highly responsive to two TLR7 ligands, 
imiquimod and gardiquimod, in transfected HEK293T cells and Cos-7 cells $(18,22)$. However, a combination of poly (dT) ODN plus the hTLR8 ligand, R848, could activate mTLR8 and induce the transcription of NF- $\kappa \mathrm{B}$ and the secretion of TNF- $\alpha$ in HEK 293 cells and PBMCs derived from mice (7). In the present study, it was also demonstrated that the overexpression of mTLR8 activated NF- $\kappa$ B and induced TNF- $\alpha$ in HEK293T cells. Additionally, a previous report demonstrated that VACV and its poly(A)/T-rich DNA motifs are potent inducers of pDC-derived IFN- $\alpha$ in TLR9-independent, and exclusively TLR8-dependent, pathways (8). However, another previous study provided evidence that poly $(\mathrm{A}) / \mathrm{T}$-rich DNA caused no activation of NF- $\kappa \mathrm{B}$ and TNF- $\alpha$ in the TLR8-dependent pathway (9). This controversy is not only because DNA has not been previously linked to TLR8 activation, but also because murine pDCs are generally known to express TLR7 and TLR9, and not TLR8. Therefore, future studies are required to elucidate the precise expression of mTLR8 mRNA in pDCs and cDCs and the functions of mTLR8 in the induction of NF- $\mathrm{BB}$ activation and in TNF- $\alpha$ or other cytokine production with combinations of poly(dT) ODN and R848, or poly(A)/T-rich DNA.

In conclusion, the present study cloned the ORF sequence of MTLR8 and analyzed the sequence signature, secondary structure and crystal structure, as well as the evolutionary association among mammalian species. The expression profile of mTLR8 in different tissues was also demonstrated. It was also confirmed that mTLR8 is functional and involved in the innate immune signaling pathways to contribute to the activation of $N F-\kappa B$ and the induction of TNF- $\alpha$. Clarification of the role of mTLR8 in innate immune recognition system is important for it development as a model for the human tumor and autoimmune diseases.

\section{Acknowledgments}

The present study was funded by the National Natural Science Funds (nos. 31302072, 31372423 and 30871884) from the National Natural Science Foundation of China.

\section{References}

1. Wu JR and Chen ZJ: Innate immune sensing and signaling of cytosolic nucleic acids. Annu Rev Immunol 32: 461-488, 2014

2. He XB, Jia HJ, Jing ZZ and Liu DX: Recognition of pathogen-associated nucleic acids by endosomal nucleic acid-sensing toll-like receptors. Acta Biochim Biophys Sin (Shanghai) 45: 241-258, 2013.

3. Unterholzner L: The interferon response to intracellular DNA: Why so many receptors? Immunobiology 218: 1312-1321, 2013.
4. Botos I, Segal DM and Davies DR: The structural biology of toll-like receptors. Structure 19: 447-459, 2011.

5. Lim KH and Staudt LM: Toll-like receptor signaling. Cold Spring Harb Perspect Biol 5: a011247, 2013.

6. Cervantes JL, Weinerman B, Basole C and Salazar JC: TLR8: The forgotten relative revindicated. Cell Mol Immunol 9: 434-438, 2012.

7. Gorden KK, Qiu XX, Binsfeld CC, Vasilakos JP and Alkan SS: Cutting edge: Activation of murine TLR8 by a combination of imidazoquinoline immune response modifiers and polyT oligodeoxynucleotides. J Immunol 177: 6584-6587, 2006.

8. Martinez J, Huang X and Yang Y: Toll-like receptor 8-mediated activation of murine plasmacytoid dendritic cells by vaccinia viral DNA. Proc Natl Acad Sci USA 107: 6442-6447, 2010.

9. Bauer S, Bathke B, Lauterbach H, Pätzold J, Kassub R, Luber CA, Schlatter B, Hamm S, Chaplin P, Suter M and Hochrein H: A major role for TLR8 in the recognition of vaccinia viral DNA by murine pDC? Proc Natl Acad Sci USA 107: E139, 2010.

10. Demaria O, Pagni PP, Traub S, de Gassart A, Branzk N, Murphy AJ, Valenzuela DM, Yancopoulos GD, Flavell RA and Alexopoulou L: TLR8 deficiency leads to autoimmunity in mice. J Clin Invest 120: 3651-3662, 2010.

11. Ma Y, Haynes RL, Sidman RL and Vartanian T: TLR8: An innate immune receptor in brain, neurons and axons. Cell Cycle 6: 2859-2868, 2007.

12. Chuang TH and Ulevitch RJ: Cloning and characterization of a sub-family of human toll-like receptors: hTLR7, hTLR8 and hTLR9. Eur Cytokine Netw 11: 372-378, 2000.

13. Heil F, Hemmi H, Hochrein H, Ampenberger F, Kirschning C, Akira S, Lipford G, Wagner H and Bauer S: Species-specific recognition of single-stranded RNA via toll-like receptor 7 and 8. Science 303: 1526-1529, 2004.

14. Jurk M, Heil F, Vollmer J, Schetter C, Krieg AM, Wagner H, Lipford G and Bauer S: Human TLR7 or TLR8 independently confer responsiveness to the antiviral compound R-848. Nat Immunol 3: 499, 2002.

15. Hemmi H, Kaisho T, Takeuchi O, Sato S, Sanjo H, Hoshino K, Horiuchi T, Tomizawa H, Takeda K and Akira S: Small anti-viral compounds activate immune cells via the TLR7 MyD88-dependent signaling pathway. Nat Immunol 3: 196-200, 2002.

16. Ohto U, Tanji H and Shimizu T: Structure and function of toll-like receptor 8. Microbes Infect 16: 273-282, 2014.

17. Liu J, Xu C, Hsu LC, Luo YP, Xiang R and Chuang TH: A five-amino-acid motif in the undefined region of the TLR8 ectodomain is required for species-specific ligand recognition. Mol Immunol 47: 1083-1090, 2010.

18. Zhu J, Lai K, Brownile R, Babiuk LA and Mutwiri GK: Porcine TLR8 and TLR7 are both activated by a selective TLR7 ligand, imiquimod. Mol Immunol 45: 3238-3243, 2008.

19. Ziegler-Heitbrock L: The CD14+ CD16+ blood monocytes: Their role in infection and inflammation. J Leukocyte Biol 81: 584-592, 2007.

20. Alexopoulou L, Desnues B and Demaria O: Toll-like receptor 8: The awkward TLR. Med Sci (Paris) 28: 96-102, 2012 (In French)

21. Edwards AD, Diebold SS, Slack EM, Tomizawa H, Hemmi H, Kaisho T, Akira S and Reis e Sousa C: Toll-like receptor expression in murine DC subsets: Lack of TLR7 expression by CD8 alpha+ DC correlates with unresponsiveness to imidazoquinolines. Eur J Immunol 33: 827-833, 2003.

22. Zhu JZ, Brownlie R, Liu Q, Babiuk LA, Potter A and Mutwiri GK: Characterization of bovine Toll-like receptor 8: Ligand specificity, signaling essential sites and dimerization. Mol Immunol 46: 978-990, 2009. 